\title{
A NEW METHOD TO EXTRACT PERMANENT SCATTERERS
}

\author{
Yonghui KANG*, Yonghong ZHANG, Hong'an WU \\ Chinese Academy of Surveying and Mapping, Beijing 100830, China
}

KEY WORDS: Time Series InSAR; Surface Deformation Monitoring; Permanent Scatterers; Point Density;

\begin{abstract}
:
At present, time series InSAR technology has been widely used in surface deformation monitoring. The extraction of permanent scatterers is an important part, which is directly related to the accuracy of monitoring results. The existing permanent scatterers extraction methods are mainly based on the amplitude information or the coherence information, there is a problem that the point quality and point density cannot be taken into account, and the selection of the point parameters requires operators to have a wealth of experience. In order to solve the above problems, a permanent scatterers extraction method based on the combination of amplitude and model coherence coefficient is proposed in this paper. This method examines not only the amplitude information of the permanent scatterers, but also the phase information of the point. And the phase quality directly affects the accuracy of deformation inversion. This paper takes Yupu Bridge, Yiqiao Town, Xiaoshan District, Hangzhou City as the experimental area to carry out comparative experiments. The results show that the final point target density extracted by this method is 1.97 times that of the conventional method based on amplitude information, and shows the details of deformation distribution of Yupu Bridge more completely.
\end{abstract}

\section{INTRODUCTION}

With the acceleration of urbanization, the continuous rise of all kinds of tall buildings, as well as the construction of various large and medium-sized bridges, it is urgent to monitor the surface deformation of urban and key buildings. Various disasters such as road collapse, house wall cracking and urban waterlogging caused by surface deformation can be seen in all kinds of news reports from time to time. At present, the commonly used surface deformation monitoring methods mainly include bedrock bench marks, layered marks, GNSS, level points and other measurement methods, but these methods can only obtain some discrete point deformation information, but cannot get the complete deformation information in the study area, so the cost is high, and the accuracy of GNSS method to obtain vertical displacement components is relatively low (Li Deren et al., 2000). At present, time series InSAR technology is widely used in surface deformation monitoring, which has the characteristics of low cost, wide coverage and area monitoring. The time series InSAR surface deformation monitoring techniques mainly include: least square method (Lundgren P et al., 2001; Usai S \& Klees R, 2002), Permanent Scatterers Interferometry (PS-InSAR) method (Ferretti A et al., 1999; Ferretti A et al., 2000; Ferretti A, 2001), Small Baseline Subset Interferometry(SBAS InSAR) method (Berardino P et al., 2002; Berardino P et al., 2003; Berardino P et al., 2003; Lanari $\mathrm{R}$ et al., 2004) and Coherent Targets method (Mora O et al., 2003a; Mora O et al.,2003b). Time series InSAR technology has been widely used in volcanoes, earthquakes, landslides, land subsidence and other fields.

In all the methods mentioned above, the permanent scatterers needs to be extracted first, and then the subsequent deformation inversion needs to be carried out. At present, the main point selection methods include: (1) selecting based on amplitude information (HOOPER A, 2008), this method uses the amplitude information of time series to select permanent scatterers, but requires at least 25 SAR images, and the threshold setting needs to be determined according to experience; (2) based on the selection of coherence coefficient (Shuning, 1999), this method uses the interference coherence of master and slave images as the screening criterion of permanent scatterers, which will be affected by the spatial-temporal baseline decoherence and the shadow response of window size in the process of calculating coherence coefficient (Touzi R. \& Lopez A, 1999). The same threshold setting needs to be determined by experience. (3) Comprehensive selection of multiple attributes, including the combination of coherence coefficient threshold method and amplitude deviation threshold method (Chen Qiang, 2006). Based on amplitude information, coherence coefficient, amplitude deviation information threelevel detection method (Hu Bo et al., 2011), "three-threshold two-stage detection method" (Jiang Decai et al., 2017), these three methods are a combination of amplitude information and coherence information. There is still a problem of threshold setting. The three-stage semi-automatic permanent scatterers detection method (Fan Xuejiao, 2016) is also a combination of amplitude information and coherence information. Although it is no longer necessary to set the threshold manually, it turns the problem of threshold setting into the selection of PS dominant area and non-PS dominant area, but there is still a problem of manual interpretation.

In 2011, Sousa $\mathbf{J}$ et al. made a comparative analysis of the two point selection methods of DePSI (Delft PSI processing package) and StaMPS (Stanford Method for Persistent Scatterers), and briefly described the principles of the two point selection methods. In the process of DePSI point selection strategy, it is necessary to remove the atmospheric phase and then encrypt the combined amplitude of the first-level point targets. As for the StaMPS point selection strategy, the point density is low in conventional cases, so oversampling can greatly improve the point selection density, but this will introduce some unstable points, which need to be reprocessed later. At the same time, the iterative process is involved in the two selection strategies, which ensures the quality and density

Corresponding author: Kang Yonghui, E-mail: kangyh@casm.ac.cn 
of the selection, but greatly increases the time cost of the selection.

The point density of these point selection methods cannot meet the monitoring of bridges or tall buildings. In view of the above problems, a point selection method based on amplitude and model coherence coefficient is proposed in this paper. This method takes into account the quality and density of point targets in the research area, and can get better results for operators without too much experience. Its main feature is that it examines not only the amplitude information or coherence information of the point target, but also the phase information of the point, and the quality of the phase information directly affects the accuracy of the deformation inversion results, and the time cost of point selection is not very high.

\section{POINT SELECTION METHOD}

One of the characteristics of the permanent scatterers is that the phase is almost stable in the time series SAR image. The phase standard deviation can be used to determine whether the phase of the permanent scatterers is stable or not. However, it is difficult to obtain the phase standard deviation in practice. Therefore, in 2001, Ferretti et al. proposed to use amplitude deviation instead of phase standard deviation to screen stable point targets, on the basis of which further research was carried out in this paper.

Firstly, the candidate points of permanent scatterers are obtained by preliminary screening based on amplitude deviation and average amplitude information. In this process, the amplitude deviation threshold and average amplitude threshold can be relaxed as far as possible to ensure that most of the candidate point targets are selected. In this process, the function of the average amplitude is mainly to eliminate the water covered area which cannot be eliminated by the amplitude deviation, so the average amplitude threshold should not be too high. Then the time series phase analysis of the candidate point targets obtained by the initial step screening is carried out.

In time series differential processing, the differential phase of a point can be expressed as shown in formula (1) (Zhang Yonghong et al., 2009; Wu Hongan, 2011)

$\varphi=w\left(\frac{4 \pi}{\lambda} T v+\varphi_{\text {non }}+\frac{4 \pi B_{\perp}}{\lambda R \sin (\theta)} \Delta h+\varphi_{\text {atm }}+\varphi_{\text {orb }}+\varphi_{\text {noise }}\right)$

where $\varphi=$ differential interference phase of a point

$w(\cdot)=$ phase wrapping

$\lambda=$ radar wavelength

$T=$ temporal baseline of differential interferogram

$v=$ linear deformation velocity

$\varphi_{\text {non }}=$ nonlinear deformation phase

$B_{\perp}=$ perpendicular baseline of the differential

interferogram

$R=$ slant range

$\theta=$ angle of incidence

$\Delta h=$ error of DEM

$\varphi_{\text {atm }}=$ atmospheric phase

$\varphi_{\text {orb }}=$ phase caused by orbital error

$\varphi_{\text {noise }}=$ the phase of out-of-coherence noise
The Delaunay triangulation is constructed for the candidate point targets, and the model phase on the edge can be obtained by the differential processing of the two candidate point targets on each edge.

$$
\varphi_{\mathrm{mod} e l}=\frac{4 \pi}{\lambda} T \Delta v+\frac{4 \pi B_{\perp}}{\lambda R \sin \theta} \Delta h^{\prime}
$$

where $\Delta v=$ the velocity increment on each edge

$\Delta h^{\prime}=$ the elevation increment on each edge

Based on this model phase, the following objective function is constructed to solve these two parameters, and the optimization is used to solve these two parameters.

$$
\gamma_{\text {model }}=\frac{1}{M}\left|\sum_{i=1}^{M} \exp \left[\mathrm{j}\left(\delta \varphi_{i}-\delta \varphi_{\bmod e l, i}\right)\right]\right|
$$

where $\mathrm{M}=$ the number of interferograms

$\gamma_{\text {model }}=$ model coherence coefficient, the range of values is $[0,1]$.

The optimal solutions for velocity and elevation increments are reached when the model coherence reaches its maximum value. Considering that the formula is used to solve the deformation velocity and elevation error later, this method is also used to screen the candidate points for primary selection. The mode coherence coefficient can well reflect whether the phase of the two points on the edge of the strip is stable or not.

The Delaunay triangulation is constructed for the candidate points, and the model coherence coefficient of each edge is calculated. After all the edges are solved, the edges whose model coherence coefficient is greater than a certain threshold ( 0.5 in this paper) are selected as reliable edges, and the corresponding two endpoints are considered as real permanent scatterers. The final selected permanent scatterers are reconstructed based on the multiple images coherent targets small baselines InSAR(MCTSB-InSAR) (Zhang Yonghong et al., 2014; Yonghong Zhang et al., 2016; Wu Hongan et al., 2016) inversion of deformation information in the study area.

\section{EXPERIMENT AND RESULT ANALYSIS}

\subsection{Experimental area and experimental data}

The experimental area is Yupu Bridge, Yiqiao Town, Xiaoshan District, Hangzhou City. Because it is the monitoring of individual buildings, the Italian X-band COSMO-SkyMed highresolution radar image is selected as the experimental data. The imaging period is from January 10, 2015 to March 3, 2018, with a total of 41 images. The central incident angle of the image is $40^{\circ}$, and the azimuth resolution and slant range resolution are $1.92 \mathrm{~m}$ and $1.14 \mathrm{~m}$, respectively. The specific parameters of the images are shown in Table 1. In order to remove the topographic phase, the SRTM DEM data with $30 \mathrm{~m}$ resolution in the research area are used for differential interference processing.

\begin{tabular}{cccc}
\hline ID & Date & $\begin{array}{c}\text { Temporal } \\
\text { Baseline(day) }\end{array}$ & $\begin{array}{c}\text { Perpendicular } \\
\text { Baseline }(\mathrm{m})\end{array}$ \\
\hline 1 & 20150110 & 0 & 0.00 \\
2 & 20150211 & 32 & 77.18 \\
3 & 20150311 & 60 & -135.92 \\
4 & 20150412 & 92 & -1288.84 \\
5 & 20150514 & 124 & -1259.94 \\
6 & 20150615 & 156 & -418.23
\end{tabular}




\begin{tabular}{|c|c|c|c|}
\hline 7 & 20150717 & 188 & -751.35 \\
\hline 8 & 20150818 & 220 & -322.22 \\
\hline 9 & 20150919 & 252 & -783.34 \\
\hline 10 & 20151021 & 284 & -346.24 \\
\hline 11 & 20151122 & 316 & -456.33 \\
\hline 12 & 20151224 & 348 & -1125.60 \\
\hline 13 & 20160109 & 364 & -308.40 \\
\hline 14 & 20160301 & 416 & -214.17 \\
\hline 15 & 20160329 & 444 & -18.02 \\
\hline 16 & 20160430 & 476 & -447.58 \\
\hline 17 & 20160516 & 492 & -63.83 \\
\hline 18 & 20160719 & 556 & 292.69 \\
\hline 19 & 20160804 & 572 & -995.37 \\
\hline 20 & 20160909 & 608 & -1175.73 \\
\hline 21 & 20160925 & 624 & 44.31 \\
\hline 22 & 20161011 & 640 & -280.48 \\
\hline 23 & 20161027 & 656 & 208.56 \\
\hline 24 & 20161128 & 688 & 369.55 \\
\hline 25 & 20161210 & 700 & -283.88 \\
\hline 26 & 20161230 & 720 & 307.37 \\
\hline 27 & 20170212 & 764 & -630.99 \\
\hline 28 & 20170228 & 780 & -132.00 \\
\hline 29 & 20170316 & 796 & -72.93 \\
\hline 30 & 20170401 & 812 & -888.47 \\
\hline 31 & 20170421 & 832 & -452.58 \\
\hline 32 & 20170523 & 864 & 231.44 \\
\hline 33 & 20170726 & 928 & -303.61 \\
\hline 34 & 20170811 & 944 & -891.70 \\
\hline 35 & 20170908 & 972 & -775.33 \\
\hline 36 & 20171014 & 1008 & -852.10 \\
\hline 37 & 20171115 & 1040 & -756.73 \\
\hline 38 & 20171217 & 1072 & -399.03 \\
\hline 39 & 20180118 & 1104 & -672.75 \\
\hline 40 & 20180130 & 1116 & -936.78 \\
\hline 41 & 20180303 & 1148 & -638.54 \\
\hline
\end{tabular}

Table 1. COSMO-SkyMed image parameters used in this paper

\subsection{Experimental results and analysis}

First of all, determine the small baseline pair according to the spatio-temporal baseline threshold, and check whether the generated differential interferogram has serious incoherence, eliminate the seriously incoherent differential interferogram, and ensure the final network connectivity. In this experiment, a total of 106 differential interferograms are generated, and the spatial-temporal baseline distribution of interference pairs is shown in figure 1.

Then the candidate points of the permanent scatterers in the study area are extracted according to the amplitude deviation and the average amplitude, the amplitude deviation threshold and the average amplitude threshold are set to 0.45 and 0.5 respectively, a total of 24810 point targets are selected, and the Delaunay triangulation is constructed by using the selected points. According to formula (3), the model coherence coefficient on each edge is calculated, and the edge whose model coherence coefficient is greater than 0.5 is retained, and the total number of point targets retained is 13924 . Based on the MCTSB-InSAR method, the final selected point targets are used to calculate the deformation velocity, and the final deformation results are shown in figure 2 , with a total of 13026 point targets. The reference point is indicated by the black asterisk in the figure 2 .

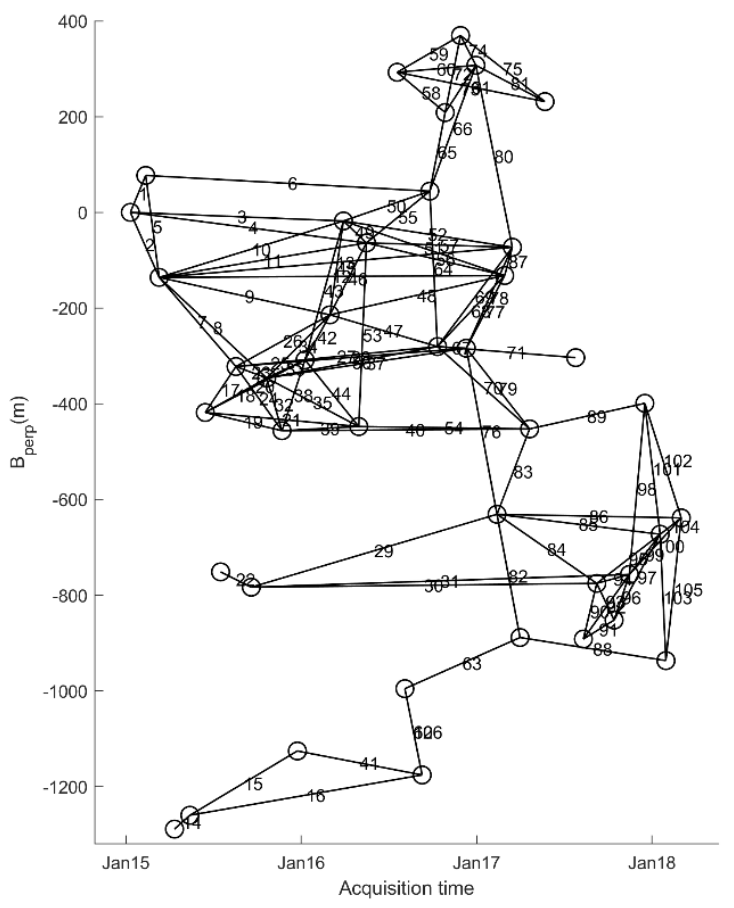

Figure 1. The spatial-temporal baseline distribution of the 106 interferograms

It can be found from figure 2 that there is obvious subsidence of the approach bridge on both sides of the Yupu Bridge, because the approach bridge does not have a very deep foundation and is directly built on the ground, the land subsidence will lead to the subsidence of the approach bridge, and the part of the bridge body is supported by piers. And the piers have a very deep foundation, so the stability of the whole bridge body is ensured. In the experimental area, the maximum deformation velocity reaches $-42 \mathrm{~mm} / \mathrm{year}$, and the deformation velocity of the bridge body is $-1 \sim 1 \mathrm{~mm} /$ year. In addition, the buildings on both sides of the approach bridge also have different degrees of subsidence.

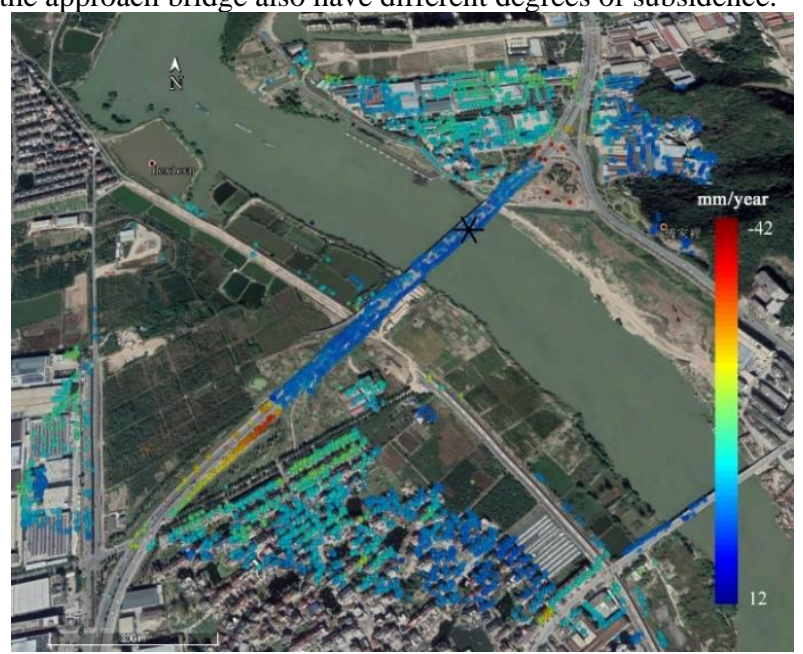

Figure 2. In this paper, the point selection method is used to obtain the deformation velocity

A comparative experiment was carried out to select points in this area based on conventional amplitude information. The amplitude deviation threshold and average amplitude threshold are set to 0.33 and 1.2 respectively to ensure the quality of point target selection and reduce the influence of pseudo points on the final deformation velocity. 6742 permanent scatterers are 
obtained. Using this point target to calculate the deformation velocity in this area, as shown in figure 3 , there are a total of 6604 permanent scatterers. The position of the reference point is the same as in figure 2 , the maximum deformation velocity is$38 \mathrm{~mm} /$ year, and the deformation velocity of the bridge is$1 \sim 1 \mathrm{~mm} /$ year.

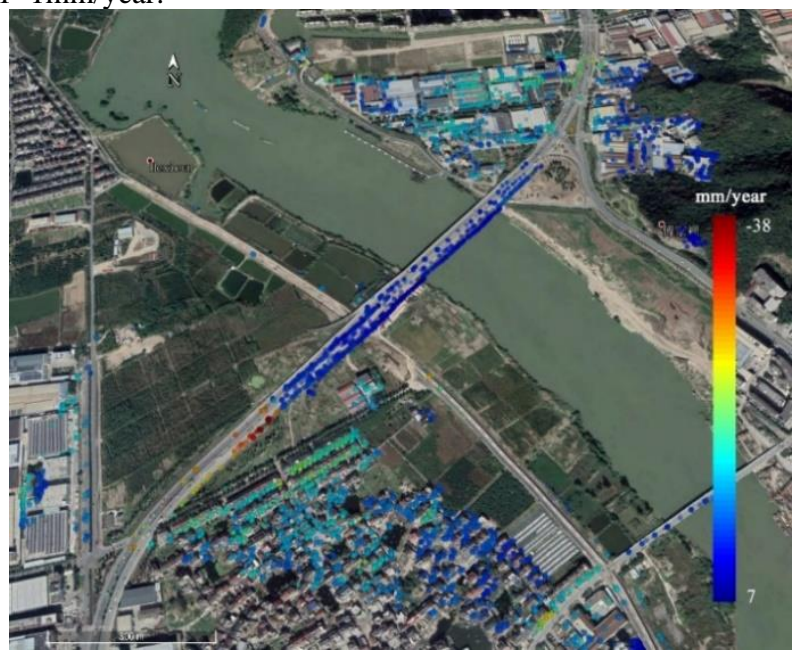

Figure 3. The deformation velocity is obtained by selecting points based on amplitude information.

By comparing figure 2 with figure 3 , we can see that the overall subsidence of the study area is basically the same, but the point density of the deformation result obtained by selecting points based on amplitude information decreases obviously, and the number of point targets from the whole bridge body to the approach bridge decreases. The most prominent is that the approach bridge on the north side of the bridge basically has no point target. The number of point targets of the south approach bridge is also significantly lower than that obtained by the point selection method in this paper, and it is obvious that the result cannot fully display the subsidence information of the whole bridge.

At the same time, the deformation velocity difference diagram of the same PS points between the method in this paper and the result of point selection based on amplitude information is calculated. There are a total of 5950 same PS points, and the dynamic range is- $8 \sim 9 \mathrm{~mm} / \mathrm{year}$, as shown in figure 4 . It can be seen from the diagram that the difference between the two is not very great, and the maximum settlement area on the south side of the bridge is slightly different, and the reason for this difference is different from the density of the surrounding points, resulting in different network construction, and finally the calculated deformation rate is different. On the whole, the two are basically the same, only the point density of this method is higher, which further illustrates the effectiveness of this method.

In order to further verify the effect of the point selection method in this paper, the threshold setting is relaxed when using the conventional method, so that the final deformation velocity result has a higher point selection density. In order to compare the final deformation velocity results, a total of 24810 point targets selected in this paper are used for deformation inversion, and finally 13246 point targets are obtained, as shown in figure 5. The position of the reference point is the same as in figure 2 .

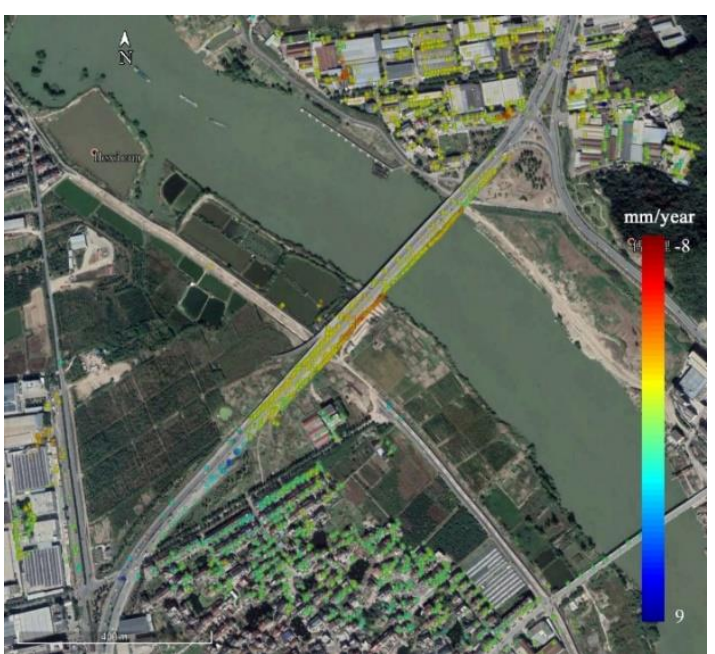

Figure 4. The deformation velocity difference diagram of the same PS points between the method in this paper and the result of point selection based on amplitude information

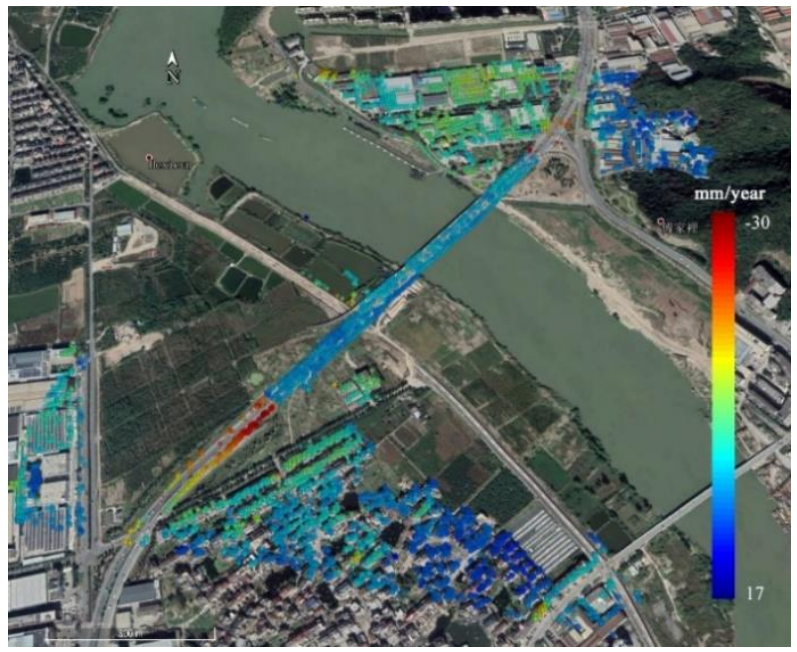

Figure 5 . The deformation velocity obtained from the primary selection points

Comparing figure 2, figure 3 and figure 5 , it can be found that although the point density of the primary point is increased, the overall deformation of the study area has changed greatly, and the deformation on both sides of the approach bridge of Yupu Bridge still exists, and the maximum deformation velocity is only $-30 \mathrm{~mm} / \mathrm{year}$, and the deformation velocity of the bridge body is $-1 \sim 1 \mathrm{~mm} /$ year. The point targets of the approach bridge on the north side of the bridge is less than that of this method. At the same time, the deformation of the bridge body is not completely consistent, and the deformation of the north side of the bridge body is obviously larger than that of the south side of the bridge body. However, the bridge itself is a rigid structure, at the same time, the bridge itself is also supported by piers with deeper foundation, so inconsistent deformation should not occur.

In addition, there are obvious differences between the southeast and northwest sides of Yupu Bridge in figure 2 and figure 5. In figure 2, there is obvious deformation in these two areas, but in figure 5 , the deformation is not obvious, and the obvious jump phenomenon occurs, and there is an uplift phenomenon in the northeast and southeast side of the bridge, which shows that although relaxing the point selection threshold increases the point density, but the deformation result is not reliable. 
The difference of the deformation velocity of the same PS points between the point selection result of this method and the initial point result is calculated, as shown in figure 6 . The total number of same PS points is 12372 . It can be seen from the figure that the range of the difference between them is-13 $4 \mathrm{~mm} / \mathrm{year}$, and the difference of the bridge basically fluctuates near zero, while there is an obvious difference between the northeast side and the south side of the bridge. the main reason for this difference is that too many noise points in the initial selection lead to calculation errors.

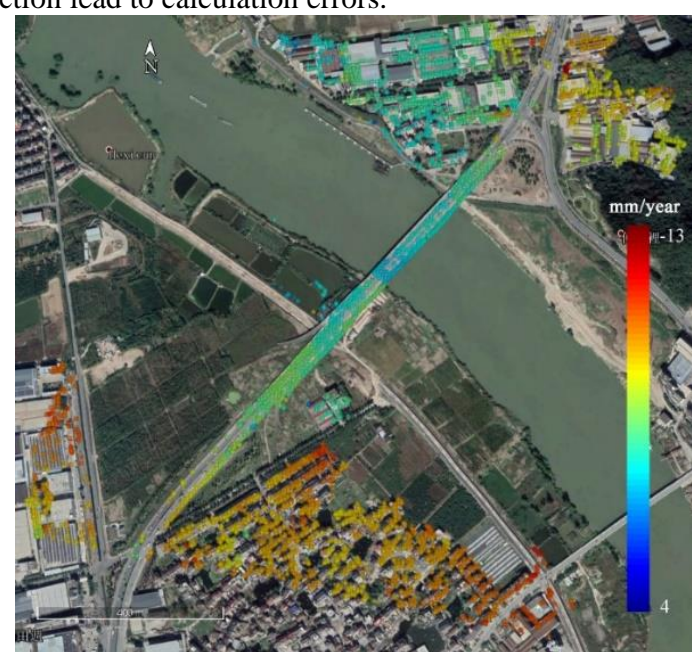

Figure 6 . The deformation velocity difference diagram of the same points between the method in this paper and the initial point selection method

\section{CONCLUSION}

In this paper, a point selection method based on amplitude and model coherence coefficient is proposed. This method not only ensures the point density, but also ensures the correctness of the final deformation velocity results. Taking the Yupu Bridge in Yiqiao Town, Xiaoshan District, Hangzhou as the experimental object, the comparative experimental results show that the final point targets density extracted by this method is about 1.97 times based on the amplitude information, and can more completely obtain the deformation details of the bridge. It shows that the method in this paper is feasible and effective.

\section{ACKNOWLEDGEMENTS}

The authors would like to thank E-geos and Beijing Vastitude Technology Co.,Ltd for providing the COSMO-SkyMed SAR images used in this paper. We also thank USGS for supporting the SRTM DEM used in this research

Financial assistance: National Key R\&D Program of China(2017YFE0107100, 2018YFB0505400); National Natural Science Foundation of China(41874014)

\section{REFERENCES}

Berardino P., Casu F., Fornaro G., et al. Small baseline DInSAR technique for earth surface deformation analysis. FRING2003, Franscati, Italy.

Berardino P., Fornaro G., Lanari R., 2002. A new algorithm for surface deformation monitoring base on small baseline differential interferograms. IEEE Transactions on Geoscience and Remote Sensing,40(11):2375-2383. doi: 10.1109/TGRS.2002.803792 · Source: IEEE Xplore.
Berardino P., Fornaro G., Lanari R., et al., 2003. A two-scale differential SAR interferometry approach for investigating earth surface deformations. IGARSS, 1184-1186. doi: 10.1109/IGARSS.2003.1294052.

Chen Q., 2006. Detecting regional ground deformation by differential SAR interferometry based on permanent scatterer. Shanghai: Southwest Jiaotong University.

Fan X. J., 2016. Study of Stable Point Targets Detection and its Application. Beijing: China Academy of surveying and Mapping.

Ferretti A., Prati C., Rocca F., 2000. Nonlinear subsidence rate estimation using the permanent scatterers in differential SAR interferometry. IEEE Transaction on Geoscience and Remote Sensing, 38(5): 2202-2212. doi: 10.1109/36.868878 - Source: IEEE Xplore.

Ferretti A., Prati C., Rocca F., 2001. Permanent scatterers in SAR interferometry. IEEE transactions on geosciences and remote sensing, 39(1):8-20. DOI: 10.1109/36.898661 - Source: IEEE Xplore.

Ferretti A., Rocca F., Prati C., 1999. Permanent scatterers in SAR interferometry. IGARSS, Hamburg Germany.

HOOPER A. J., 2008. A Multi-temporal InSAR Method Incorporating Both Persistent Scatterer and Small Baseline Approaches. Geophysical Research Letters, 35(16): 96-106. doi:10.1029/2008GL034654

Hu B., Wang H. S., Jia L., 2011. Automatic detection of permanent scatterers in PSInSAR. Science of surveying and Mapping, 36 (2): 51- 52. doi: 10.16251/j.cnki.10092307.2011.02.047.

Jiang D. C., Zhang Y. H., Zhang J. X., et al., 2017. Uneven Land Subsidence Along Tianjin Subway Lines Monitored by InSAR Technology. Remote Sensing Information, 32 (6): 2732. DOI: 10.3969/j.issn.1000-3177.2017.06.005

Lanari R., Mora O., Manunta M., et al.,2004. A small-baseline approach for investigating deformations on full-resolution differential SAR interferograms. IEEE Transactions on Geoscience and Remote Sensing,42(7):1377-1386. DOI: 10.1109/TGRS.2004.828196 · Source: IEEE Xplore.

Li D. R., Zhou Y. Q., Ma H. C., 2000. Principles and Applications of Interferometry SAR. Science of surveying and Mapping,20 (1): 9-12.

Lundgren P., Usai S., Sansonsti E., 2001. Modeling surface deformation observed with synthetic aperture radar interferometry at Campi Flegrei caldera. Journal of Geophysical Research, 106:19355-19366. DOI: 10.1029/2001JB000194.

Mora O., Mallorqui J. J., Broquetas A., 2003a. Linear and nonlinear terrain deformation maps from a reduced set of interferometric SAR images. IEEE Transactions on Geoscience and Remote Sensing,41 (10):2243-2252. DOI: 10.1109/TGRS.2003.814657 · Source: IEEE Xplore.

Mora O., Mallorqui J. J., Lanari R.,2003b. Differential interferometric SAR techniques for generation of terrain and building deformation maps. Proceedings of de la 5a Setmana Geomatica de Barcelone. 
Shu N., 1999: Principles of Microwave Remote Sensing. Wuhan University Press, Wuhan.

Sousa J., Hooper A. J., Hanssen R., et al., 2011, Persistent Scatterer InSAR: A comparison of methodologies based on a model of temporal deformation vs. spatial correlation selection criteria. Remote Sensing of Environmen,115: 2652-2663. DOI:10.1016/j.rse.2011.05.021

Touzi R., Lopez A., 1999. Coherence estimation for SAR imagery. IEEE trans. Geosci. Remote Sensing, 37(1):135-149. DOI: 10.1109/36.739146 · Source: IEEE Xplore.

Usai S., Klees R., 2002. SAR interferometry on a very long time scale: a study of the interferometric characteristics of manmade features. IEEE Transactions on Geoscience and Remote Sensing, 40(11):2375-2383. DOI: 10.1109/36.774730 - Source: IEEE Xplore.

Wu H. A., Zhang Y. H., Chen X. Y., et al., 2011. Ground deformation monitoring using smallbaseline DlnSAR teehnique:A case study inTaiyuan City from 2003 to 2009. Chinese Journal of Geophysics,54 (3): 673-680. DOI:10.3969/J.issn.0001-5733.2011.03.006.

Wu H. A., Zhang Y. H., Kang Y. H., et al., 2016. A Fast Multilayer Subnetwork Connection Method for Time Series InSAR Technique. Journal of surveying and Mapping,45 (10): 11921199. DOI: 10.11947/j.AGCS.2016.20160033.

Zhang Y. H., Wu H. A., Kang Y. H., et al., 2016. Ground Subsidence in the Beijing-Tianjin-Hebei Region from 1992 to 2014 Revealed by Multiple SAR Stacks. Remote Sensing, 8(675): 1 -17. DOI: $10.3390 /$ rs 8080675

Zhang Y. H., Wu H. A., Zhang L. M., et al., 2014. Traffic network settlement monitoring based on high resolution time series satellite SAR images. Advances in Geodesy and Geodynamics: part II.

Zhang Y. H., Zhang J. X., Gong W. Y., et al., 2009. Monitoring Urban Subsidence Based on SAR Interferometric Point Target Analysis. Journal of surveying and Mapping,38 (6): 482$487,493$. 